\title{
Additive Conservation Laws in Local Relativistic Quantum Field Theory
}

\author{
W. D. Garber and H. Reeh \\ Institut für Theoretische Physik, D-3400 Göttingen, Federal Republic of Germany
}

\begin{abstract}
We consider generators $Q$ of symmetry transformations acting additively on asymptotic particle states according to (1.1). [This equation can be derived for $Q$ defined as integral over a conserved local current!]. For simplicity, we consider only the case that all asymptotic fields are scalar. Assuming that elastic scattering occurs at least in an open subset of the scattering manifold we show that $Q$ is at most a linear combination of generators of the Poincaré group and internal symmetries.
\end{abstract}

\section{Introduction}

A couple of investigations led to the conclusion that in a quantum field theory with sufficient interaction, there are no other symmetries apart from internal ones and those of the Poincaré group. Important contributions were [1] and [2]. In [1] the conclusion was obtained by assuming in particular the $S$-matrix to be analytic in the whole physical region and the symmetry generators to be self-adjoint. In [2], the conclusion was proven for a subclass of translation invariant generators.

In the present paper we take up this problem within the Wightman framework augmented by rather modest additional assumptions. Clearly, some kind of interaction assumption is necessary: It is known from examples that in theories without interaction there are many more generators than those mentioned above, see e.g. [5]. Furthermore, if generators are defined as integrals over conserved local hermitian current densities, there is an example showing that such a generator need not have self-adjoint extensions [5]. Hence, a hypothesis of selfadjointness should be avoided.

Considering a generator $Q$ as an integral over a conserved local not necessarily covariant current density, we proved in $[3,4]$ that

$$
i\left[Q, \psi_{\kappa}^{\mathrm{ex}}(x)\right]=P_{\kappa \lambda}(x, \partial) \psi_{\lambda}^{\mathrm{ex}}(x)
$$

(Summation convention!). Here, $\psi_{\kappa}^{\mathrm{ex}}(x)$ ("ex" stands for "in" or "out") are free asymptotic fields, $P_{\kappa \lambda}$ are polynomials in $x \in \mathbb{R}^{4}$ and derivatives $\partial=\left(\frac{\partial}{\partial x^{v}}\right)$, 
vanishing for unequal masses $M_{\kappa} \neq M_{\lambda}$. Note that $P_{\kappa \lambda}$ does not depend on the index "ex" so that $Q$ commutes with the $S$-matrix. This was obtained by assuming

(i) existence of a mass gap above the vacuum,

(ii) isolated one-particle hyperboloids of finite multiplicity,

(iii) invariance of the vacuum,

(iv) asymptotic completeness,

(v) existence of a local interpolating field for every particle.

Starting from (1.1), we showed in [5] that $Q$ on one-particle states with positive mass, i.e. $\left\{P_{\kappa \lambda}\right\}_{\kappa \lambda}$, is a polynomial in generators of the Poincaré group with matrices as coefficients operating within the different mass multiplets. Therefore, in case all asymptotic fields are scalar fields $\Phi_{\kappa}^{\text {ex }},(1.1)$ can be written as

$$
i\left[Q, \Phi_{\kappa}^{\mathrm{ex}}(x)\right]=R_{\kappa \lambda}\left(x_{v} \partial_{\mu}-x_{\mu} \partial_{v}, \partial_{\varrho}\right) \Phi_{\lambda}^{\mathrm{ex}}(x)
$$

with polynomials $R_{\kappa \lambda}$ vanishing for $M_{\kappa} \neq M_{\lambda}$.

It is our aim to show that $P_{\kappa \lambda}$ is at most a linear combination of the generators of the Poincare group and internal symmetries. Concerning the interaction, we assume within every mass multiplet:

i) For each particle there is another particle such that elastic scattering occurs on some open subset $V$ of the set of momenta allowed by energy and momentum conservation.

ii) The mass multiplet does not decompose into subsets of particles with no interaction between members of different subsets.

The open subset $V$ may depend on the pair of particles.

In the following, we present a proof for the case that all asymptotic fields are scalar, i.e., we prove the corresponding statement for $R_{\kappa \lambda}$. Since $R_{\kappa \lambda}$ vanishes for $M_{\kappa} \neq M_{\lambda}$, we need only`consider one mass multiplet.

\section{Case of Translation Invariant Generators}

In the present section, we consider the case of translationally invariant generators for which (1.2) reads

$$
i\left[Q, \Phi_{\kappa}^{\mathrm{ex}}(x)\right]=R_{\kappa \lambda}(\partial) \Phi_{\lambda}^{\mathrm{ex}}(x) .
$$

The general case will be proved by reduction to this case in Sect. 3. To illustrate part of the method, we treat first the simplest case:

\subsection{One Scalar Field}

We consider a theory with only one incoming and one outgoing real field $\Phi^{\mathrm{ex}}(x)$ for the mass multiplet under consideration. Two such particles may give rise to elastic scattering. The set of momenta allowed by energy and momentum conservation will be called scattering manifold $\mathscr{M}$,

$$
\begin{aligned}
\mathscr{M}:= & \left\{\left(\mathbf{p}_{1}, \mathbf{p}_{2}, \mathbf{p}_{3}, \mathbf{p}_{4}\right) \in \mathbb{R}^{12} \mid \mathbf{p}_{1}+\mathbf{p}_{2}=\mathbf{p}_{3}+\mathbf{p}_{4}, \omega\left(\mathbf{p}_{1}\right)+\omega\left(\mathbf{p}_{2}\right)=\omega\left(\mathbf{p}_{3}\right)+\omega\left(\mathbf{p}_{4}\right),\right. \\
& \left.\omega(\mathbf{p}):=\sqrt{\mathbf{p}^{2}+M^{2}}\right\} .
\end{aligned}
$$


Lemma 2.1. Let $Q$ be a generator with

$$
i\left[Q, \Phi^{\mathrm{ex}}(x)\right]=R(\partial) \Phi^{\mathrm{ex}}(x) .
$$

Assume that there is scattering (i.e. $S \neq 1$ ) on some open subset $V$ of the scattering manifold $\mathscr{M}$. Then one obtains

$$
R(\partial)=a^{v} \partial_{v}, \quad a^{v} \in \mathbb{C} .
$$

Proof. i) We need only consider hermitian $Q$. Then $R$ on the mass shell is real. It is odd there since from the Jacobi identity

$$
\begin{aligned}
{\left[\left[Q, \Phi^{\mathrm{ex}}(x)\right], \Phi^{\mathrm{ex}}(y)\right] } & =\left[\left[Q, \Phi^{\mathrm{ex}}(y)\right], \Phi^{\mathrm{ex}}(x)\right], \\
R\left(\partial_{x}\right) \Delta(x-y) & =-R\left(-\partial_{x}\right) \Delta(x-y) .
\end{aligned}
$$

ii) We denote the Fourier transformed fields by $\tilde{\Phi}^{\mathrm{ex}}(p)$ and the vacuum by $\Omega$. From

$$
\begin{aligned}
& \left(Q \tilde{\Phi}^{\mathrm{in}}\left(p_{1}\right) \tilde{\Phi}^{\mathrm{in}}\left(p_{2}\right) \Omega \mid \tilde{\Phi}^{\mathrm{out}}\left(p_{3}\right) \tilde{\Phi}^{\mathrm{out}}\left(p_{4}\right) \Omega\right) \\
& \quad=\left(\tilde{\Phi}^{\mathrm{in}}\left(p_{1}\right) \tilde{\Phi}^{\mathrm{in}}\left(p_{2}\right) \Omega \mid Q \tilde{\Phi}^{\mathrm{out}}\left(p_{3}\right) \tilde{\Phi}^{\mathrm{out}}\left(p_{4}\right) \Omega\right)
\end{aligned}
$$

one gets using (2.1) and i)

$$
\begin{aligned}
& \left(R\left(i p_{1}\right)+R\left(i p_{2}\right)-R\left(i p_{3}\right)-R\left(i p_{4}\right)\right) \\
& \quad \cdot\left(\tilde{\Phi}^{\mathrm{in}}\left(p_{1}\right) \tilde{\Phi}^{\mathrm{in}}\left(p_{2}\right) \Omega \mid \tilde{\Phi}^{\mathrm{out}}\left(p_{3}\right) \tilde{\Phi}^{\mathrm{out}}\left(p_{4}\right) \Omega\right)=0 .
\end{aligned}
$$

The second factor is a distribution with support in $\mathscr{M}$. By assumption, this distribution is non-zero in $V$. Putting

$$
R(i \mathbf{p}, i \omega(\mathbf{p}))=: f(\mathbf{p})
$$

we get

$$
f\left(\mathbf{p}_{1}\right)+f\left(\mathbf{p}_{2}\right)-f\left(\mathbf{p}_{3}\right)-f\left(\mathbf{p}_{4}\right)=0, \quad\left(\mathbf{p}_{1}, \mathbf{p}_{2}, \mathbf{p}_{3}, \mathbf{p}_{4}\right) \in V .
$$

iii) Now consider (2.4) in the lab system, $\mathbf{p}_{2}=0$. We want to show that (2.4) extends to the whole scattering manifold in the lab system $\mathscr{M}_{L} . f(\mathbf{p})$ is real analytic. For given $\mathbf{p}_{1}$, the allowed $\mathbf{p}_{3}, \mathbf{p}_{4}=\mathbf{p}_{1}-\mathbf{p}_{3}$ lie on the ellipsoid (Fig. 1)

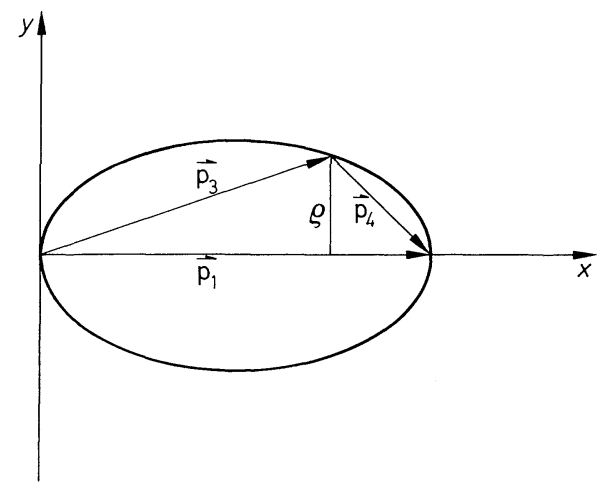

Fig. 1. Scattering manifold 
with half-axes [6]

$$
\begin{aligned}
& a=\frac{1}{2}\left|\mathbf{p}_{1}\right| \\
& b=\left|\mathbf{p}_{1}\right|\left(2\left(1+\frac{\omega\left(\mathbf{p}_{1}\right)}{M}\right)\right)^{1 / 2} .
\end{aligned}
$$

We parametrize the ellipsoid by $\mathbf{p}_{3}=\mathbf{p}_{3}\left(\mathbf{p}_{1}, \beta, \psi\right) ; 0 \leqq \beta<\infty ; 0 \leqq \psi<2 \pi$ as follows :

$$
\begin{aligned}
& \left(p_{3}\right)_{x}=\frac{2}{1+\beta^{2}} a \\
& \left(p_{3}\right)_{y}=\varrho(\beta) \cos \psi, \quad \varrho(\beta)=\frac{2 \beta}{1+\beta^{2}} \cdot b \\
& \left(p_{3}\right)_{z}=\varrho(\beta) \sin \psi .
\end{aligned}
$$

For $\mathbf{p}_{1} \neq 0,0<\beta<\infty, 0 \leqq \psi<2 \pi, \mathbf{p}_{3}$ is real analytic in $\mathbf{p}_{1}, \beta, \psi$.

For $\mathbf{p}_{2}=0$, the left hand side of (2.4) is analytic on $\mathscr{M}_{L} \backslash\left\{\mathbf{p}_{1}=0\right\}$ and hence vanishes there. By continuity, (2.4) holds on all of $\mathscr{M}_{L}$.

iv) The validity of (2.4) on $\mathscr{M}_{L}$ would imply by a known theorem [7] that $f$ and thus $R$ is a linear combination of $\mathbf{p}$ and $\omega(\mathbf{p})$. The constant term vanishes by (2.3). This would finish the proof. In fact, a simplified version of the quoted theorem will suffice here, a short proof of which will be reported in the appendix.

\subsection{Several Scalar Fields}

Extending a result of Lopuszanski [2], we now consider the general case of (2.1). In momentum space, $(2.1)$ reads

$$
i\left[Q, \tilde{\Phi}_{\kappa}^{\mathrm{ex}}(p)\right]=R_{\kappa \lambda}(i p) \tilde{\Phi}_{\lambda}^{\mathrm{ex}}(p) .
$$

A Jacobi identity similar to (2.3) yields on the mass shell

$$
R_{\kappa \lambda}(i p)=-R_{\lambda \kappa}(-i p) .
$$

We again may assume that $Q$ is real. In case $R_{\kappa \lambda}$ is a homogeneous polynomial it follows that $R_{\kappa \lambda}$ is symmetric or antisymmetric in the indices and can be diagonalized by a unitary transformation of one-particle states which, however, is $p$-dependent in general and then useless. For special cases, this complication does not occur:

2.2. Lemma. Let $Q$ be a generator with

$$
i\left[Q, \tilde{\Phi}_{\kappa}^{\mathrm{ex}}(p)\right]=r_{\kappa \lambda} R(i p) \tilde{\Phi}_{\lambda}^{\mathrm{ex}}(p),
$$

where the p-independent $r_{\kappa \lambda}$ is symmetric or antisymmetric and $R$ is a polynomial. Assume that there is elastic scattering, for the mass multiplet considered, in the following sense: For each particle $\varrho$ there is another particle $\tau$ such that elastic scattering occurs on some open subset $V_{\varrho \tau}$ of the scattering manifold. Then on the mass shell

$$
r_{\kappa \lambda} R(i p)=\mathbf{a}_{\kappa \lambda} \mathbf{p}+a_{\kappa \lambda}^{0} \omega(\mathbf{p})+c_{\kappa \lambda} .
$$


If furthermore the mass multiplet does not decompose into subsets of particles with no interaction between members of different subsets, then

$$
r_{\kappa \lambda} R(i p)=\delta_{\kappa \lambda}\left(\mathbf{a p}+a^{0} \omega(\mathbf{p})\right)+c_{\kappa \lambda} .
$$

Remarks. i) The interaction assumption is strong in the sense that it must hold for every basis of one particle states.

ii) The assumption is weak in the sense that $V_{\varrho \tau}$ may vary with the pair of particles considered.

Proof. i) We diagonalize $r_{\kappa \lambda}$ by a basis transformation mapping $\left\{\Phi_{\lambda}^{\mathrm{ex}}\right\}$ onto $\left\{\hat{\Phi}_{\lambda}^{\mathrm{ex}}\right\}$ so that

$$
i\left[Q, \tilde{\hat{\Phi}}_{\lambda}^{\mathrm{ex}}(p)\right]=r_{\lambda} R(i p) \tilde{\hat{\Phi}}_{\lambda}^{\mathrm{ex}}(p)
$$

(no summation in $\lambda$ !). Considering now

$$
\left(\tilde{\hat{\Phi}}_{\varrho}^{\mathrm{out}}\left(p_{1}\right) \tilde{\hat{\Phi}}_{\tau}^{\mathrm{out}}\left(p_{2}\right) \Omega \mid Q \tilde{\hat{\Phi}}_{\varrho}^{\mathrm{in}}\left(p_{3}\right) \tilde{\hat{\Phi}}_{\tau}^{\mathrm{in}}\left(p_{4}\right) \Omega\right)
$$

and proceeding as in the proof of Lemma 2.1, we arrive at an equation corresponding to $(2.4)$

$$
f_{\varrho}\left(\mathbf{p}_{1}\right)+f_{\tau}\left(\mathbf{p}_{2}\right)-f_{\varrho}\left(\mathbf{p}_{3}\right)-f_{\tau}\left(\mathbf{p}_{4}\right)=0, \quad\left(p_{1}, p_{2}, p_{3}, p_{4}\right) \in V_{\varrho \tau}
$$

with

$$
f_{\varrho}(\mathbf{p}):=r_{\varrho} R(i \mathbf{p}, i \omega(\mathbf{p})) .
$$

In the lab system, e.g. for particle $\tau\left(\mathbf{p}_{2}=0\right)$, we can as before analytically continue (2.8) to all of $\mathscr{M}_{L}$. By the appendix,

$$
f_{i}(\mathbf{p})=\mathbf{a}_{i} \mathbf{p}+a_{i}^{0} \omega(\mathbf{p})+c_{i}, \quad i=\varrho, \tau,
$$

with

$$
\mathbf{a}_{\varrho}=\mathbf{a}_{\tau}, \quad a_{\varrho}^{0}=a_{\tau}^{0},
$$

where $\mathbf{a}_{i}, a_{i}^{0}, c_{i}$ are numbers. Returning to the original basis, one gets the first statement.

ii) With the stronger interaction assumption, choosing a suitable sequence of index pairs one gets that $\mathbf{a}_{i}, a_{i}^{0}$ now do not depend on the index $i$.

The aim is next to reduce the case of general $Q$ to the previous lemma. The idea is to separate the terms in $R$ of highest degree and show that they are linear by using Lemma 2.2.

2.3. Theorem. Let $Q$ be a translation invariant generator

$$
i\left[Q, \tilde{\Phi}_{\kappa}^{\mathrm{ex}}(p)\right]=R_{\kappa \lambda}(i p) \tilde{\Phi}_{\lambda}^{\mathrm{ex}}(p)
$$

with polynomials $R_{\kappa \lambda}$ vanishing for $M_{\kappa} \neq M_{\lambda}$. Consider one mass multiplet (mass $M$ ) and assume that there is scattering in the following sense:

i) For each particle @ there is another particle $\tau$ such that elastic scattering occurs on some open subset $V_{\varrho \tau}$ of the scattering manifold. 
ii) The mass multiplet does not decompose into subsets of particles with no interaction between members of different subsets. Then

$$
R_{\kappa \lambda}(i p)=\delta_{\kappa \lambda}\left(\mathbf{a p}+a^{0} \omega(\mathbf{p})\right)+c_{\kappa \lambda}
$$

(a, $a^{0}$ may depend on the mass multiplet).

(Concerning the interaction assumptions, note also the remarks following Lemma 2.2.)

Remark. If contrary to assumption ii) there are subsets with no interaction between particles of different subsets, assumption i) implies (2.9) only on the subsets (a, $a^{0}$ may be different on different subsets).

Proof. i) We rewrite (2.5)

$$
\begin{aligned}
i\left[Q, \tilde{\Phi}_{\kappa}^{\mathrm{ex}}(p)\right] & =a_{\kappa \lambda}^{n_{0} n_{1} n_{2} n_{3}} p_{0}^{n_{0}} p_{1}^{n_{1}} p_{2}^{n_{2}} p_{3}^{n_{3}} \tilde{\Phi}_{\lambda}^{\mathrm{ex}}(p), \quad n_{0} \in\{0,1\} \\
& =\left(b_{\kappa \lambda}^{n_{1} n_{2} n_{3}}+p_{0} c_{\kappa \lambda}^{n_{1} n_{2} n_{3}}\right) p_{1}^{n_{1}} p_{2}^{n_{2}} p_{3}^{n_{3}} \tilde{\Phi}_{\lambda}^{\mathrm{ex}}(p) .
\end{aligned}
$$

Define the set of coefficients

$$
A:=\left\{a_{\kappa \lambda}^{n_{0} n_{1} n_{2} n_{3}} \neq 0 \mid M_{\kappa}=M_{\lambda}=M\right\}
$$

and three subsets

$$
A_{i}:=\left\{a_{\kappa \lambda}^{n_{0} n_{1} n_{2} n_{3}} \in A \mid n_{0}+n_{i} \text { maximal }\right\}, \quad i=1,2,3 .
$$

The maximal value $n_{0}+n_{i}$ occurring in $A_{i}$ will be called $d_{i}$. Choose a set $A_{k}$ with highest $d_{k}$. By a choice of coordinates, let $k=1$. For $d_{1}=0,(2.9)$ follows. So suppose $d_{1} \geqq 1$. Then $A_{1}$ has elements $b_{\kappa \lambda}^{N_{1}, n_{2}, n_{3}}, c_{\kappa \lambda}^{N_{1}-1, n_{2}, n_{3}}$ with $n_{2}, n_{3} \leqq N_{1}, N_{1}:=d_{1}$.

ii) We want to separate the terms of highest degree in (2.5) by boosts. Apply to (2.5) a Lorentz transformation

$$
U(\Lambda) \tilde{\Phi}_{\kappa}^{\mathrm{ex}}(p) U^{-1}(\Lambda)=\tilde{\Phi}_{\kappa}^{\mathrm{ex}}(\Lambda p)
$$

to get for the Lorentz transformed generator

$$
i\left[U(\Lambda) Q U^{-1}(\Lambda), \tilde{\Phi}_{\kappa}^{\mathrm{ex}}(p)\right]=R_{\kappa \lambda}\left(i \Lambda^{-1} p\right) \tilde{\Phi}_{\lambda}^{\mathrm{ex}}(p) .
$$

For a boost $\Lambda_{1}^{-1}(\alpha)$ along the $p_{1}$-axis,

$$
\begin{aligned}
& \left(\Lambda_{1}^{-1}(\alpha) p\right)_{0}=p_{0} \operatorname{ch} \alpha+p_{1} \operatorname{sh} \alpha \\
& \left(\Lambda_{1}^{-1}(\alpha) p\right)_{1}=p_{0} \operatorname{sh} \alpha+p_{1} \operatorname{ch} \alpha,
\end{aligned}
$$

we consider

$$
Q^{\alpha}=(\operatorname{ch} \alpha)^{-N_{1}} U\left(\Lambda_{1}(\alpha)\right) Q U^{-1}\left(\Lambda_{1}(\alpha)\right) .
$$

In the corresponding polynomials

$$
R_{\kappa \lambda}^{\alpha}(i p)=(\operatorname{ch} \alpha)^{-N_{1}} R_{\kappa \lambda}\left(i \Lambda_{1}^{-1}(\alpha) p\right),
$$

for $\alpha \rightarrow \infty$ only the highest-order terms will survive, more precisely the terms with coefficients in $A_{1}$ :

$$
R_{\kappa \lambda}^{\infty}(i p)=\lim _{\alpha \rightarrow \infty} R_{\kappa \lambda}^{\alpha}(i p)=\left(b_{\kappa \lambda}^{N_{1} n_{2} n_{3}}+c_{\kappa \lambda}^{N_{1}-1, n_{2} n_{3}}\right)\left(p_{0}+p_{1}\right)^{N_{1}} p_{2}^{n_{2}} p_{3}^{n_{3}}
$$

with summation over $n_{2}, n_{3}$ only. 
iii) Just as $Q$ is defined by $R_{\kappa \lambda}$, the polynomials $R_{\kappa \lambda}^{\infty}$ define an operator $Q^{\infty}$ with $i\left[Q^{\infty}, \tilde{\Phi}_{\kappa}^{\mathrm{ex}}(p)\right]=R_{\kappa \lambda}^{\infty}(i p) \tilde{\Phi}_{\lambda}^{\mathrm{ex}}(p)$.

To prove (2.10), define operators $i\left(Q^{\infty}\right)^{\text {ex }}$ by

$$
\int R_{\kappa \lambda}^{\infty}(i \mathbf{p} ; i \omega(\mathbf{p})) a_{\kappa}^{\mathrm{ex}^{\dagger}}(\mathbf{p}) a_{\lambda}^{\mathrm{ex}}(\mathbf{p}) \frac{d^{3} \mathbf{p}}{2 \omega(\mathbf{p})}
$$

with canonical creation and annihilation operators belonging to the asymptotic fields. Then

$$
\begin{aligned}
& i\left[\left(Q^{\infty}\right)^{\mathbf{e x}}, a_{\sigma}^{\mathbf{e x}^{\dagger}}(\mathbf{q})\right]=R_{\kappa \sigma}^{\infty}(i \mathbf{q}, i \omega(\mathbf{q})) a_{\kappa}^{\mathrm{ex}^{\dagger}}(\mathbf{q}) \\
& i\left[\left(Q^{\infty}\right)^{\mathrm{ex}}, a_{\sigma}^{\mathrm{ex}}(\mathbf{q})\right]=-R_{\sigma \lambda}^{\infty}\left(i \mathbf{q}, i \omega(\mathbf{q}) a_{\lambda}^{\mathbf{e x}}(\mathbf{q}) .\right.
\end{aligned}
$$

From (2.6) we have for the limit polynomial also that

$$
R_{\kappa \lambda}^{\infty}(i p)=-R_{\lambda \kappa}^{\infty}(-i p) .
$$

Hence,

$$
i\left[\left(Q^{\infty}\right)^{\mathrm{ex}}, \tilde{\Phi}_{\kappa}^{\mathrm{ex}}(p)\right]=R_{\kappa \lambda}^{\infty}(i p) \tilde{\Phi}_{\lambda}^{\mathrm{ex}}(p) .
$$

Now $Q$ commutes with $S$ as does $U(\Lambda)$ and therefore $Q^{\alpha}$. As a form, $Q^{\alpha}$ converges to $\left(Q^{\infty}\right)^{\text {ex }}$. Therefore, also $Q^{\infty}$ commutes with $S$, hence $\left(Q^{\infty}\right)^{\text {in }}=\left(Q^{\infty}\right)^{\text {out }}=: Q^{\infty}$ and (2.10) holds.

iv) $Q^{\infty}$ needs further decomposition. As a first step, we successively apply boosts $\Lambda_{2}\left(\alpha_{2}\right), \Lambda_{3}\left(\alpha_{3}\right)$ in the $p_{2}, p_{3}$-directions. In the polynomials $R_{\kappa \lambda}^{\infty}$, call $N_{2}$ the highest degree in $p_{2}$ and consider

$$
\left(\operatorname{ch} \alpha_{2}\right)^{-N_{1}-N_{2}} U\left(\Lambda_{2}(\alpha)\right) Q^{\infty} U^{-1}\left(\Lambda_{2}\left(\alpha_{2}\right)\right)
$$

which in the limit $\alpha_{2} \rightarrow \infty$, by repeating the arguments of (ii), (iii), yields an operator $Q^{\infty, 2}$ with

$$
i\left[Q^{\infty, 2}, \tilde{\Phi}_{\kappa}^{\mathrm{ex}}(p)\right]=\left(b_{\kappa \lambda}^{N_{1} N_{2} n_{3}}+c_{\kappa \lambda}^{N_{1}-1, N_{2}, n_{3}}\right)\left(p_{0}+p_{2}\right)^{N_{1}+N_{2}} p_{3}^{n_{3}} \tilde{\Phi}_{\lambda}^{\mathrm{ex}}(p)
$$

where the sum is only over $n_{3}$.

Call $N_{3}$ the highest degree of the remaining polynomial, and consider

$$
\left(\operatorname{ch} \alpha_{3}\right)^{-N_{1}-N_{2}-N_{3}} U\left(\Lambda_{3}\left(\alpha_{3}\right)\right) Q^{\infty, 2} U^{-1}\left(\Lambda_{3}\left(\alpha_{3}\right)\right) .
$$

In the limit, this defines an operator $Q^{\infty, 3}$ with

$$
i\left[Q^{\infty, 3}, \tilde{\Phi}_{\kappa}^{\mathrm{ex}}(p)\right]=\left(b_{\kappa \lambda}^{N_{1} N_{2} N_{3}}+c_{\kappa \lambda}^{N_{1}-1, N_{2}, N_{3}}\right)\left(p_{0}+p_{3}\right)^{N_{1}+N_{2}+N_{3}} \tilde{\Phi}_{\lambda}^{\mathrm{ex}}(p)
$$

with no summation over $N_{1}, N_{2}, N_{3}$.

Applying (2.6) to the polynomials in (2.11), it follows that $b_{\kappa \lambda}^{N_{1} N_{2} N_{3}}+c_{\kappa \lambda}^{N_{1}-1, N_{2}, N_{3}}$ is either symmetric or antisymmetric in $\kappa, \lambda$. By Lemma $2.2\left(N_{1} \geqq 1\right.$ by assumption, see above!)

$$
\left(b_{\kappa \lambda}^{N_{1} N_{2} N_{3}}+c_{\kappa \lambda}^{N_{1}-1, N_{2}, N_{3}}\right)\left(\omega(\mathbf{p})+p_{3}\right)^{N_{1}+N_{2}+N_{3}}=\delta_{\kappa \lambda}\left(\mathbf{a p}+a^{0} \omega(\mathbf{p})\right) .
$$

Hence, $N_{2}=N_{3}=0, N_{1}=1$, and $b_{\kappa \lambda}^{100}+c_{\kappa \lambda}^{000}$ is independent of $\kappa, \lambda$. 
v) By applying a rotation with $p_{1} \rightarrow-p_{1}$ to the original $Q$ and following the steps i) to iv), we see that $-b_{\kappa \lambda}^{100}+c_{\kappa \lambda}^{000}$ is independent of $\kappa, \lambda$. Thus, both coefficients are independent of $\kappa, \lambda$ separately.

From iv), $1=N_{1}=d_{1}$. By definition, $d_{1} \geqq d_{2} \geqq d_{3} \geqq 0$. Hence the original polynomial $R_{\kappa \lambda}$ is at most linear. To see that the remaining coefficients $b_{\kappa \lambda}^{010}, b_{\kappa \lambda}^{001}$ are independent of $\kappa, \lambda$, take different sequences of boosts and proceed as above.

\section{General Case}

In [5] it was shown that for a general generator $Q$, the polynomials $P_{\kappa \lambda}\left(x, \partial_{x}\right)$ can be written as polynomials in the generators $M_{\mu \nu}, P_{\varrho}$ of the Poincare group. For this result, we explicitly used $M^{2}>0$ thereby excluding the generators of the conformal group $\left(M^{2}>0\right.$, of course, was also used in deriving (1.1) in [3] by application of Haag-Ruelle scattering theory). This $M^{2}>0$ assumption will again be used in the following. The simplest non-translation invariant generators are:

\subsection{Generators of First Order in $M_{\mu \nu}$}

In this case,

$$
i\left[Q, \Phi_{\kappa}^{\mathrm{ex}}(x)\right]=\left\{\left(x_{v} \partial_{\mu}-x_{\mu} \partial_{\nu}\right) d_{\kappa \lambda}^{\mu \nu}(\partial)+d_{\kappa \lambda}(\partial)\right\} \Phi_{\lambda}^{\mathrm{ex}}(x)
$$

with polynomials $d_{\kappa \lambda}, d_{\kappa \lambda}^{\mu v}=-d_{\kappa \lambda}^{v \mu}$. Application of an infinitesimal translation leads to a translation invariant generator $Q^{\varrho}:=i\left[P^{\varrho}, Q\right]$ and

$$
i\left[Q^{\varrho}, \Phi_{\kappa}^{\mathrm{ex}}(x)\right]=2 d_{\kappa \lambda}^{\varrho \mu}(\partial) \partial_{\mu} \Phi_{\lambda}^{\mathrm{ex}}(x) .
$$

Theorem 2.3 leads to

$$
d_{\kappa \lambda}^{\varrho \mu}(i p) p_{\mu}=\delta_{\kappa \lambda} a^{\varrho \mu} p_{\mu}+b_{\kappa \lambda}^{\varrho}
$$

on the mass shell. We now need an auxiliary lemma:

3.1. Lemma. For polynomials $d^{v \mu}($ ip $)$ with $d^{v \mu}=-d^{\mu v}$ and

$$
d^{\varrho \mu}(i p) p_{\mu}=a^{\varrho \mu} p_{\mu}+b^{\varrho}
$$

on $p^{2}=M^{2}$ it follows that $a^{\varrho \mu}=-a^{\mu \varrho}$ and $b^{\varrho}=0$.

Proof. Since $d^{\varrho \mu}$ is antisymmetric,

$$
a^{\varrho \mu} p_{\mu} p_{\varrho}+b^{\varrho} p_{\varrho}=0
$$

on $p^{2}=M^{2}$. Explicitly, with Latin indices running from 1 to 3 ,

$$
\begin{aligned}
& \omega(\mathbf{p})\left\{\left(a^{0 i}+a^{i 0}\right) p_{i}+b^{0}\right\} \\
& \quad+\left\{\left(a^{i k}+a^{k i}\right) p_{i} p_{k}+b^{i} p_{i}+a^{00}\left(\mathbf{p}^{2}+M^{2}\right)\right\}=0 .
\end{aligned}
$$

The curly brackets have to vanish since otherwise $\omega(\mathbf{p})$ would be a rational function, however $\omega(\mathbf{p})=\sqrt{\mathbf{p}^{2}+M^{2}}$. Since $M^{2}>0$, equating equal powers yields the statement. 
Application to (3.2) gives $b_{\kappa \lambda}^{\varrho}=0$, and

$$
\left(d_{\kappa \lambda}^{\varrho \mu}(i p)-\delta_{\kappa \lambda} a^{\varrho \mu}\right) p_{\varrho}=0
$$

on $p^{2}=M^{2}$, where both terms in the bracket are antisymmetric in $\varrho, \mu$. Inserting (3.3) into (3.1), we get

$$
i\left[Q, \Phi_{\kappa}^{\mathrm{ex}}(x)\right]=\left\{\left(x_{\nu} \partial_{\mu}-x_{\mu} \partial_{\nu}\right) \delta_{\kappa \lambda} a^{\mu \nu}+d_{\kappa \lambda}(\partial)\right\} \Phi_{\lambda}^{\mathrm{ex}}(x) .
$$

Since $M_{\mu \nu}$ clearly is a symmetry generator, $Q-a^{\mu v} M_{\mu \nu}$ is a generator too, with $i\left[Q-a^{\mu v} M_{\mu \nu}, \Phi_{\kappa}^{\mathrm{ex}}(x)\right]=d_{\kappa \lambda}(\partial) \Phi_{\lambda}^{\mathrm{ex}}(x)$

and hence is translationally invariant. Theorem 2.3 leads to

$$
d_{\kappa \lambda}(i p)=\delta_{\kappa \lambda} a^{v} p_{v}+c_{\kappa \lambda}
$$

on $p^{2}=M^{2}$.

We have proven:

3.2. Lemma. Let $Q$ be a generator fulfilling (3.1). With the interaction assumptions i), ii) of Theorem 2.3, one has

$$
i\left[Q, \Phi_{\kappa}^{\mathrm{ex}}(x)\right]=\left\{\delta_{\kappa \lambda}\left(\left(x_{\nu} \partial_{\mu}-x_{\mu} \partial_{\nu}\right) a^{v \mu}+a^{v} \partial_{\nu}\right)+c_{\kappa \lambda}\right\} \Phi_{\lambda}^{\mathrm{ex}}(x) .
$$

\subsection{Generators of Arbitrary Order in $M_{\mu \nu}$}

In [5] it was shown that an arbitrary generator can be put into the form

$$
i\left[Q, \Phi_{\kappa}^{\mathrm{ex}}(x)\right]=\sum_{n=0}^{N} x_{v_{1}} \partial_{\mu_{1}} \ldots x_{v_{n}} \partial_{\mu_{n}} d_{\kappa \lambda}^{\left(v_{1} \mu_{1}\right) \ldots\left(v_{n} \mu_{n}\right)}(\partial) \Phi_{\lambda}^{\mathrm{ex}}(x)
$$

with polynomials $d$ antisymmetric under the exchange of $v_{i}$ with $\mu_{i}$ for the same $i$, and symmetric under permutation of $\left(v_{i}, \mu_{i}\right)$ with $\left(v_{k}, \mu_{k}\right)$.

We show that terms with $N \geqq 2$ do not occur. Assume $N \geqq 2$. Then (3.6) can be rewritten

$$
i\left[Q, \Phi_{\kappa}^{\mathrm{ex}}(x)\right]=\left\{x_{v_{1}} \ldots x_{v_{N}} \partial_{\mu_{1}} \ldots \partial_{\mu_{N}} d_{\kappa \lambda}^{\left(v_{1} \mu_{1}\right) \ldots\left(v_{N} \mu_{N}\right)}+\ldots\right\} \Phi_{\lambda}^{\mathrm{ex}}(x),
$$

where the dots denote terms of order less than $N$ in $x$. Consider

$$
Q^{\varrho_{2} \ldots \varrho_{N}}:=i\left[P^{\varrho_{2}}, i\left[P^{\varrho_{3}}\left[\ldots i\left[P^{\varrho_{N}}, Q\right] \ldots\right]\right]\right.
$$

for which

$$
i\left[Q^{\varrho_{2} \ldots \varrho_{N}}, \Phi_{\kappa}^{\mathrm{ex}}\right]=\left\{(N-1) ! x_{v_{1}} \partial_{\mu_{1}} \partial_{\mu_{2}} \ldots \partial_{\mu_{N}} d_{\kappa \lambda}^{\left(v_{1} \mu_{1}\right)\left(\varrho_{2} \mu_{2}\right) \ldots \varrho_{N} \mu_{N}}(\partial)+\ldots\right\} \Phi_{\lambda}^{\mathrm{ex}}(x),
$$

where the dots now denote polynomials in $\partial$ only. Lemma 3.2 implies

$$
p_{\mu_{2}}\left(p_{\mu_{3}} \ldots p_{\mu_{N}} d_{\kappa \lambda}^{\left(\nu_{1} \mu_{1}\right)\left(\varrho_{2} \mu_{2}\right) \ldots\left(\varrho_{N} \mu_{N}\right)}(i p)\right)=\delta_{\kappa \lambda} a^{\nu_{1} \mu_{1} \varrho_{2} \ldots \varrho_{N}} .
$$

We apply Lemma 3.1 to $p_{\mu_{3}} \ldots p_{\mu_{N}} d_{\kappa \lambda}^{\left(v_{1} \mu_{1}\right)\left(\varrho_{2} \mu_{2}\right) \ldots\left(\varrho_{N} \mu_{N}\right)}$ which is antisymmetric in $\mu_{2}$, $\varrho_{2}$ to get $a^{v_{1} \mu_{1} \varrho_{2} \ldots \varrho_{N}}=0$. Reinserting (3.8) with $a=0$ into (3.7) shows that the term of order $N$ vanishes. 
Repeating the process, one sees that only terms of order 1 may occur. Hence we have shown

3.3. Theorem. Let $Q$ be an arbitrary generator (fulfilling (1.2)), and assume the interaction hypotheses i) and ii) of Theorem 2.3. Then

$$
i\left[Q, \Phi_{\kappa}^{\mathrm{ex}}(x)\right]=\left\{\delta_{\kappa \lambda}\left[\left(x_{\nu} \partial_{\mu}-x_{\mu} \partial_{v}\right) a^{v \mu}+a^{v} \partial_{v}\right]+c_{\kappa \lambda}\right\} \Phi_{\lambda}^{\mathrm{ex}}(x) .
$$

\section{Concluding Remarks}

i) No specific assumption of local quantum field theory has been used in showing that a $Q$ obeying (1.1) can only be a linear combination of generators of the Poincaré group and internal symmetries in its action on a mass multiplet of scalar particles with $M>0$ if there is elastic scattering in the sense of i), ii) of Theorem 2.3. In $[3,4]$ we derived (1.1) within the Wightman framework of field theory. One could alternatively take the point of view that every observable commuting with the $S$-matrix and acting additively on asymptotic states fulfils (1.2) in any local quantum field theory, and follow our line of reasoning. An assumption like (1.2) was the starting point of [8] who applied the method of [1] including supersymmetries. To avoid complications in the present paper, we did not consider the case of particles with spin nor supersymmetries.

ii) The present investigation shows that the example of a generator with no selfadjoint extension given in [5] does not occur in a theory with elastic scattering.

iii) In classical mechanics of mass points (relativistic or Newtonian) the quantities corresponding to $Q$ are additive conservation laws. The analogue of Theorem 2.3 is (A4) of the appendix. A classical analogue of Theorem 3.3 may also be proven.

\section{Appendix}

Consider two functions $f_{i}(\mathbf{p}), i=1,2, \mathbf{p} \in \mathbb{R}^{3}$ fulfilling the functional equation

$$
f_{1}\left(\mathbf{p}_{1}\right)+f_{2}(\mathbf{0})=f_{1}\left(\mathbf{p}_{3}\right)+f_{2}\left(\mathbf{p}_{4}\right)
$$

whenever

$$
\mathbf{p}_{1}=\mathbf{p}_{3}+\mathbf{p}_{4}
$$

and

$$
\omega\left(\mathbf{p}_{1}\right)+M=\omega\left(\mathbf{p}_{3}\right)+\omega\left(\mathbf{p}_{4}\right), \quad \omega(\mathbf{p})=\sqrt{\mathbf{p}^{2}+M^{2}}=: h\left(\mathbf{p}^{2}\right)
$$

i.e. on the scattering manifold $\mathscr{M}_{L}$ in the lab system of particle 2. For a rather general class of functions $f_{i}$ it follows that [7]

$$
f_{i}(\mathbf{p})=\mathbf{a p}+a^{0} \omega(\mathbf{p})+b_{i}, \quad i=1,2
$$

with constants $a, a^{0}, b_{i}$.

The following simple proof for continuously differentiable functions we learned from D. Maison. [The proof, in fact, uses (A1) only on a small subset of $\mathscr{M}_{L}$ ]: 
Inserting (A2) into (A1) and (A3),

$$
f_{1}\left(\mathbf{p}_{1}\right)+f_{2}(\mathbf{0})=f_{1}\left(\mathbf{p}_{3}\right)+f_{2}\left(\mathbf{p}_{1}-\mathbf{p}_{3}\right)
$$

whenever

$$
h\left(\mathbf{p}_{1}^{2}\right)+M=h\left(\mathbf{p}_{3}^{2}\right)+h\left(\left(\mathbf{p}_{1}-\mathbf{p}_{3}\right)^{2}\right) .
$$

Varying $\mathbf{p}_{3} \rightarrow \mathbf{p}_{3}+\delta\left(\mathbf{p}_{3}\right)$ on the manifold given by (A3'), we get from (A3')

$$
0=\delta\left(\mathbf{p}_{3}\right) \cdot\left[\mathbf{p}_{3} h^{\prime}\left(\mathbf{p}_{3}^{2}\right)-\left(\mathbf{p}_{1}-\mathbf{p}_{3}\right) h^{\prime}\left(\left(\mathbf{p}_{1}-\mathbf{p}_{3}\right)^{2}\right)\right] \text {. }
$$

For variations $\delta\left(\mathbf{p}_{3}\right)$ allowed by $\left(\mathrm{A} 3^{\prime \prime}\right)$,

$$
0=\delta\left(\mathbf{p}_{3}\right) \cdot\left(\nabla f_{1}\left(\mathbf{p}_{3}\right)-\nabla f_{2}\left(\mathbf{p}_{1}-\mathbf{p}_{3}\right)\right) .
$$

For a variation around forward scattering, $\mathbf{p}_{3}=\mathbf{p}_{1},\left(\mathrm{~A} 3^{\prime \prime}\right)$ implies

$$
0=\delta\left(\mathbf{p}_{3}\right) \cdot \mathbf{p}_{3} .
$$

Replacing $f_{i}$ by $\hat{f}_{i}(\mathbf{p})=f_{i}(\mathbf{p})-\mathbf{p} \nabla f_{2}(\mathbf{0})$ for which (A1) to (A3) still hold, we get

$$
0=\delta\left(\mathbf{p}_{3}\right) \cdot \nabla f_{1}\left(\mathbf{p}_{3}\right)
$$

for all $\delta\left(\mathbf{p}_{3}\right)$ orthogonal to $\mathbf{p}_{3}$. Hence $\hat{f}_{1}(\mathbf{p})=g_{1}\left(\mathbf{p}^{2}\right)$,

$$
f_{1}(\mathbf{p})=\mathbf{p} \nabla f_{2}(\mathbf{0})+g_{1}\left(\mathbf{p}^{2}\right)
$$

and

$$
\boldsymbol{\nabla} f_{1}(\mathbf{0})=\boldsymbol{\nabla} f_{2}(\mathbf{0}) .
$$

Similarly, by considering a variation $\mathbf{p}_{4} \rightarrow \mathbf{p}_{4}+\delta\left(\mathbf{p}_{4}\right)$ around backward scattering $\mathbf{p}_{4}=\mathbf{p}_{1}$,

$$
f_{2}(\mathbf{p})=\mathbf{p} \nabla f_{1}(\mathbf{0})+g_{2}\left(\mathbf{p}^{2}\right) .
$$

We have to show that $g_{1}=g_{2}=a^{0} h$. $\left(\mathrm{A} 1^{\prime \prime}\right)$ holds for $f_{i}$ replaced by $\hat{f}_{i}$. Inserting $\hat{f}_{i}=g_{i}$ we get

$$
0=\delta\left(\mathbf{p}_{3}\right) \cdot \mathbf{p}_{3} g_{1}^{\prime}\left(\mathbf{p}_{3}^{2}\right)-\delta\left(\mathbf{p}_{3}\right)\left(\mathbf{p}_{1}-\mathbf{p}_{3}\right) g_{2}^{\prime}\left(\left(\mathbf{p}_{1}-\mathbf{p}_{3}\right)^{2}\right) .
$$

Multiplying with $h^{\prime}\left(p_{3}^{2}\right)$ and using $\left(\mathrm{A} 3^{\prime \prime}\right)$, we arrive at

$$
0=\delta\left(\mathbf{p}_{3}\right) \cdot\left(\mathbf{p}_{1}-\mathbf{p}_{3}\right)\left(g_{1}^{\prime}\left(\mathbf{p}_{3}^{2}\right) h^{\prime}\left(\left(\mathbf{p}_{1}-\mathbf{p}_{3}\right)^{2}\right)-g_{2}^{\prime}\left(\left(\mathbf{p}_{1}-\mathbf{p}_{3}\right)^{2}\right) h^{\prime}\left(p_{3}^{2}\right) .\right.
$$

Since for $\mathbf{p}_{3} \neq \mathbf{p}_{1}$ one may choose $\delta\left(\mathbf{p}_{3}\right)$ such that $\delta\left(\mathbf{p}_{3}\right) \cdot\left(\mathbf{p}_{1}-\mathbf{p}_{3}\right) \neq 0$ (see e.g. Fig. 1$)$,

$$
\frac{g_{1}^{\prime}\left(\mathbf{p}_{3}^{2}\right)}{h^{\prime}\left(\mathbf{p}_{3}^{2}\right)}=\frac{g_{2}^{\prime}\left(\left(\mathbf{p}_{1}-\mathbf{p}_{3}\right)^{2}\right)}{h^{\prime}\left(\left(\mathbf{p}_{1}-\mathbf{p}_{3}\right)^{2}\right)} \text {. }
$$

Choosing $\mathbf{p}_{3}=\mathbf{p}_{1}$ resp. $\mathbf{p}_{3}=\mathbf{0}$ [for which (A7) holds by continuity!], one gets

$$
\begin{aligned}
& g_{1}^{\prime}\left(\mathbf{p}^{2}\right)=\frac{g_{2}^{\prime}(0)}{h^{\prime}(0)} \cdot h^{\prime}\left(\mathbf{p}^{2}\right) \\
& g_{2}^{\prime}\left(\mathbf{p}^{2}\right)=\frac{g_{1}^{\prime}(0)}{h^{\prime}(0)} \cdot h^{\prime}\left(\mathbf{p}^{2}\right) .
\end{aligned}
$$

For $\mathbf{p}=\mathbf{0}, g_{1}^{\prime}(0)=g_{2}^{\prime}(0)$ follows. Integrating, we get (A4). 
Remarks. i) The proof above works also if the two particles have different masses. [Then, of course, $f_{i}(\mathbf{p})=\mathbf{a p}+a^{0} \sqrt{\mathbf{p}^{2}+M_{i}^{2}}+c_{i}$.

ii) In case of space dimension two, (A4) holds too as well as the proof above. In case of space dimension one, however, (A4) does not follow. The scattering manifold reduces to two points, and any function $f(\mathbf{p})=f_{1}(\mathbf{p})=f_{2}(\mathbf{p})$ then fulfils (A1).

Acknowledgement. We would like to thank I. Ojima for a careful and critical reading of the manuscript, and D. Maison for the proof in the Appendix.

\section{References}

1. Coleman, S., Mandula, J.: Phys. Rev. 159, 1251-56 (1967)

2. Orzalesi, C.A., Sucher, J., Woo, C.H. : Phys. Rev. Lett. 21, 1550-1553(1968)

Lopuszanski, J.T.: J. Math. Phys. 12, 2401-12 (1971)

3. Garber, W.D., Reeh, H.: J. Math. Phys. 19, 59-66 (1978)

4. Garber, W.D., Reeh, H.: J. Math. Phys. 19, 985-986 (1978)

5. Garber, W.D., Reeh, H.: Commun. Math. Phys. 67, 179-186 (1979)

6. Landau, L.D., Lifschitz, E.M.: Lehrbuch der theoretischen Physik. II, p. 32 f. Berlin: AkademieVerlag 1966

7. Chernikoff, N.A.: Acta Physica Polonica 26, 1069-1092 (1964)

Klinker, Th.: Diplomarbeit Göttingen 1979 and references quoted therein

8. Haag, R., Lopuszanski, J.T., Sohnius, M. : Nucl. Phys. B 88, 257-274 (1975)

Communicated by H. Araki

Received June 4, 1979 